\title{
Neuroimagem de receptores D2 de dopamina na esquizofrenia
}

\author{
Rodrigo A Bressana, Valeria Biglianib e Lyn S Pilowsky ${ }^{b}$

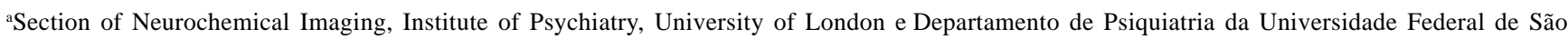 \\ Paulo (Unifesp). bection of Neurochemical Imaging, Institute of Psychiatry, University of London
}

\section{Introdução}

A esquizofrenia é uma doença de alta prevalência, afetando $1 \%$ da população ao longo da vida, principalmente indivíduos na transição da adolescência para a vida adulta, prestes a atingir o ápice do seu potencial produtivo. Geralmente tem um curso devastador, comprometendo enormemente a capacidade produtiva e social dos indivíduos afetados e causando enorme impacto nas suas famílias. A equizofrenia impõe um custo desproporcionalmente alto em relação aos demais transtornos psiquiátricos devido a gastos com hospitalização, tratamento e reabilitação, somada a perda da capacidade produtiva. ${ }^{1}$

Grandes avanços foram feitos na abordagem terapêutica da esquizofrenia. Embora novas medicações antipsicóticas e abordagens psicossociais tenham melhorado bastante o tratamento dos pacientes, ainda há muito a ser feito. Poucos são os indivíduos que voltam a ter o funcionamento compatível com as expectativas pré-mórbidas. Dos poucos que voltam a trabalhar, a maioria exerce funções muito aquém do potencial pré-mórbido.

Atualmente, já não há mais dúvida de que a esquizofrenia é um transtorno que acomete o funcionamento cerebral. Diferentes estudos vêm demonstrando alterações neuroanatômicas, neurofisiológicas e neuroquímicas, mas até o momento poucos achados resultaram em real benefício para os pacientes. Um maior entendimento da fisiopatologia da esquizofrenia possibilitará diagnósticos mais específicos, tratamentos mais eficazes e, possivelmente, prevenção. A esquizofrenia tem uma neurofisiopatologia bastante complexa, acometendo sofisticadas funções corticais, tais como linguagem, processamento do pensamento, memória e cognição. Modelos animais de investigação têm muitos problemas para simular os transtornos neurofisiológicos da esquizofrenia.

As técnicas de neuroimagem funcional e neuroquímicas têm sido consideradas o novo e mais importante paradigma de pesquisa nessa área, pois permitem avaliação in vivo. ${ }^{2}$ Tomografia por emissão de fóton único (SPET) e de pósitrons (PET), junto com ressonância magnética funcional (fMRI) e a espectroscopia por ressonância magnética (MRS), constituem as modalidades de neuroimagem mais utilizadas para estudo da esquizofrenia. As técnicas de SPET e PET permitem a avaliação in vivo dos diferentes sistemas de neurotransmissão, por meio de ligantes específicos marcados com elementos radioativos. $\mathrm{O}$ presente estudo propõe a apresentar importantes descobertas em relação a hipótese dopaminérgica da esquizofrenia e mecanismo de ação dos antipsicóticos. Para tanto, serão abordados aspectos relevantes das técnicas de imagem de neuroreceptores empregadas, as hipóteses neuroquímicas da esquizofrenia e os principais achados dos estudos. Serão discutidos o alcance e as limitações das técnicas mencionadas, assim como as perspectivas para pesquisa e as possíveis aplicações clínicas.

\section{Técnicas de imagem de neuroreceptores-SPET e PET}

O SPET e o PET são técnicas da medicina nuclear que possibilitam tanto a avaliação de neuroreceptores, como a avaliação do fluxo sangüíneo cerebral. Essas técnicas utilizam radiotraçadores, ou seja, substâncias com grande afinidade e especificidade para determinados receptores e que são marcadas com um radioisótopo. ${ }^{3}$ Durante um scan, o radiotraçador (também denominado ligante) é injetado no indivíduo e se concentra nas regiões cerebrais onde há maior número de receptores disponíveis para ligação. Com gama-câmeras, captam-se os raios gama emitidos pelo ligante. Um sistema de computador reconstrói essas informações na forma de imagens tridimensionais. As imagens finais são cortes do cérebro como numa tomografia, com a diferença de que essas imagens são mapas de distribuição e concentração do ligante no cérebro e não mapas anatômicos. Os locais em que a imagem é mais intensa correspondem às áreas em que há maior número de receptores disponíveis para interação com o ligante. A ligação do radiotraçador ao receptor depende da disponibilidade dos receptores. Substâncias endógenas (neurotransmissores) ou exógenas, que tenham afinidade pelo mesmo receptor, competem pelo sítio de ligação. Os receptores que estão ligados ao neurotransmissor endógeno não interagem com o ligante, assim como os receptores que estão ligados a qualquer outra droga, por exemplo, antipsicótico. Os radiotraçadores mais utilizados para receptores D2 de dopamina são o [ $\left.{ }^{123} \mathrm{I}\right]-$ IBZM (SPET) e $\left[{ }^{11} \mathrm{C}\right]$-raclopride (PET). Para maiores detalhes a respeito dos métodos de análise, ver os estudos de Seibyl e Bigliani \& Pilowsky. ${ }^{3,4}$

\section{Sistema dopaminérgico}

A hipótese de hiperfunção dopaminérgica continua sendo o modelo neuroquímico mais aceito para explicar a esquizofrenia. ${ }^{5}$ As duas principais fontes de evidência sustentando essa hipótese são:

1. a anfetamina (agente que induz liberação de dopamina) induz quadros psicóticos; 
2. o mecanismo de ação das drogas antipsicóticas está ligado ao bloqueio de receptores D2 de dopamina.

A anfetamina não induz sintomas negativos, portanto imagina-se que a hiperfunção do sistema dopaminérgico está mais ligada aos sintomas positivos. Além do mais, os antipsicóticos são mais efetivos para tratar sintomas positivos do que para sintomas negativos.

\section{Investigações in vivo}

\section{Densidade de receptores $D 2$}

As primeiras investigações in vivo utilizando PET datam de 1986 e sugeriam aumento do número de receptores D2 em pacientes esquizofrênicos nunca medicados. Esses achados não foram consistentemente replicados por um grande número de pesquisas que se seguiram (para revisão, ver o estudo de Bigliani \& Pilowsky). ${ }^{4}$ Sutis assimetrias na concentração de receptores D2 na região estriatal esquerda foram notados, mas pouco replicadas até o momento.

\section{Atividade dopaminérgica}

Cinco diferentes estudos avaliaram pacientes com esquizofrenia utilizando $\left[{ }^{18} \mathrm{~F}\right]$-fluorodopa ou $\left[{ }^{11} \mathrm{C}\right]$-dopa. ${ }^{6}$ Esses traçadores avaliam a atividade da enzima dopa-descarboxilase, que está envolvida na síntese de dopamina. Quatro desses estudos acharam aumento de ligação dos radiotraçadores em pacientes com esquizofrenia, quando comparados com indivíduos normais. A elevada captação desses radiotraçadores está associada a maior atividade da enzima dopa-descarboxilase. Este achado sugere aumento da atividade dopaminérgica em pacientes com esquizofrenia. Entretanto, a reação catalisada pela dopa-descarboxilase não é determinante da taxa de síntese de dopamina. Portanto, o aumento da atividade da dopadescarboxilase sugere aumento da liberação de dopamina na fenda sináptica, mas esta relação não é direta.

Laruelle et al desenvolveram um sofisticado paradigma para avaliar a atividade dopaminérgica utilizando [23 I-IBZM e SPET. ${ }^{7}$ O paradigma avalia a quantidade de receptores D2 de dopamina disponíveis antes e depois da administração de anfetamina. A administração de anfetamina induz uma liberação da dopamina, que vai se ligar aos receptores D2, diminuindo o número de receptores disponíveis para a ligação do traçador. A quantidade de dopamina liberada é estimada a partir da subtração do scan pré-anfetamina e outro scan pós-anfetamina. Os autores descobriram maior liberação de dopamina em pacientes com esquizofrenia, quando comparados aos controles normais. $\mathrm{O}$ aumento na liberação de dopamina correlacionou-se com a emergência de sintomas psicóticos. Este achado foi replicado por mais dois estudos, tanto em SPET $^{8}$ como PET. ${ }^{9}$ Com as replicações, notou-se que o aumento da responsividade do sistema dopaminérgico à anfetamina é particularmente importante nas fases de exacerbação dos sintomas psicóticos.

Abi-Dargham et al utilizaram um inibidor da tirosina-hidroxilase (metil-para-tirosina-MPT) na tentativa de mensurar a dopamina endógena em pacientes com esquizofrenia. ${ }^{6}$ A quantidade de dopamina na fenda foi estimada a partir do número de receptores
D2 disponíveis, medidos por [ $\left.{ }^{123} \mathrm{I}\right]-\mathrm{IBZM}$ antes e depois da administração da MPT. Como era esperado, a quantidade de receptores disponíveis depois da MPT aumentou, tanto nos pacientes com esquizofrenia como nos voluntários sadios. Mas o aumento de receptores disponíveis em pacientes com esquizofrenia foi significativamente maior do que nos voluntários sadios. Portanto, pacientes com esquizofrenia liberam maiores quantidades de dopamina na fenda sináptica do que indivíduos sadios. Além disso, níveis elevados de dopamina foram preditivos de boa resposta às medicações antipsicóticas.

\section{Ocupação de receptores D2 por antipsicóticos}

Todos os antipsicóticos empregados na clínica são antagonistas dos receptores D2, ou seja, ligam-se nos receptores D2 e impedem que o receptor se ligue a qualquer outra substância. Postula-se que a eficácia dessas medicações decorra deste antagonismo. Para se avaliar a intensidade do bloqueio ou ocupação dos receptores $\mathrm{D} 2 \mathrm{em}$ pacientes tomando antipsicóticos, utilizam-se basicamente duas técnicas:

1. compara-se um scan pré-tratamento com um scan póstratamento;

2. comparam-se scans de pacientes medicados com pacientes não medicados.

Essas comparações permitem inferir a porcentagem de receptores ocupados pelo antipsicótico em relação a indivíduos não medicados (Figura 1). ${ }^{5}$

Foram feitos diversos estudos com os mais diversos antipsicóticos, utilizando diferentes ligantes. Esses estudos têm contribuído para maior entendimento do mecanismo de ação das medicações antipsicóticas, tendo, portanto, importantes repercussões na prática clínica. A literatura nessa área é vasta e serão listados aqui alguns estudos, a título de ilustração.

\section{Ocupação de receptores D2 e resposta clínica}

Foi demonstrado que a ocupação de receptores D2 não é o único mecanismo responsável pela resposta clínica de pacientes com esquizofrenia. Pilowsky et al ${ }^{10}$ mostraram que pacientes que respondem a antipsicóticos típicos não apresentam ocupação de receptores D2 superior a pacientes que não respondem. Pacientes resistentes a terapêutica com antipsicóticos típicos e que respondem a clozapina, apresentam níveis de ocupação de receptores D2 (com a clozapina) inferiores aos níveis obtidos com antipsicóticos típicos. ${ }^{11}$ Apesar de não haver relação causal entre bloqueio de receptores D2 e resposta clínica aos antipsicóticos, estudos de neuroimagem mostram que a associação entre eles é inegável. Estudos prospectivos utilizando diferentes doses de antipsicóticos estimam que uma ocupação superior a $65 \%$ ou $70 \%$ é necessária para que se obtenha resposta clínica. ${ }^{12-14}$

\section{Antipsicóticos típicos versus atípicos}

Estudos de PET e $\left[{ }^{11} \mathrm{C}\right]$-raclopride demonstraram correlação positiva entre a dose do antipsicótico típico e a porcentagem de receptores ocupados. ${ }^{14,15}$ Diferentes classes de antipsicóticos típicos administrados em doses habitualmente utilizadas na clínica ocupam entre $70 \%$ e $89 \%$ dos receptores 
D2 no striatum. ${ }^{13}$ Em geral, antipsicóticos atípicos, em doses clínicas, apresentam ocupação de receptores D2 inferior aos antipsicóticos típicos (Figura 1). Tanto a clozapina como a quetiapina tendem a ocupar, no máximo, $75 \%$ dos receptores $\mathrm{D} 2$, enquanto a risperidona e a olanzapina passam a ocupar mais do que $75 \%$ dos receptores quando a dose supera $4 \mathrm{mg} \mathrm{e}$ $15 \mathrm{~g}$, respectivamente. ${ }^{15-18}$

\section{Sintomas}

Kapur et al demonstraram que, no tratamento com antipsicóticos (haloperidol), quando se atingem ocupações de receptores D2 superiores a 78\% no striatum, há grande chance dos indivíduos desenvolverem sintomas extrapiramidais. Bressan et al mostraram correlação positiva entre a ocupação de receptores D2 no striatum e a intensidade de sintomas depressivos em pacientes esquizofrênicos estáveis. Indivíduos com ocupações superiores a $78 \%$ tiveram maior chance de apresentar sintomas depressivos.*

\section{Seletividade límbica}

Os estudos reportados acima utilizaram ligantes com moderada afinidade por receptores D2 e que, portanto, conseguem discriminar somente as regiões com maior concentração de receptores D2, como o striatum. Receptores D2 localizados em áreas de grande interesse para a esquizofrenia, como o córtex temporal, não podem ser visualizados por essas técnicas. Bigliani et al utilizaram um ligante com grande afinidade por receptores D2, [ $\left.{ }^{123} \mathrm{I}\right]$-epidepride e SPET para avaliar a ocupação de receptores $\mathrm{D} 2 \mathrm{em}$ pacientes com esquizofrenia tratados com diversas doses de antipsicóticos típicos. ${ }^{19} \mathrm{O}$ ligante empregado permite tanto a visualização dos receptores D2 do striatum como do córtex temporal. Foi demonstrada que, mesmo com baixas doses, a maior parte dos receptores D2 do córtex temporal estavam ocupados. Outros estudos utilizando [ $\left.{ }^{[23} \mathrm{I}\right]$-epidepride mostraram que a clozapina, a olanzapina e a quetiapina apresentam ocupação de receptores D2 no córtex temporal superiores às encontradas no striatum..$^{20-22}$ Esse fenômeno é chamado de "seletividade límbica". Supõe-se que este seja um dos motivos porque os antipsicóticos atípicos apresentam eficácia clínica (alta ocupação temporal) com um risco menor de desencadearem sintomas extrapiramidais (baixa ocupação striatal).

\section{Conclusão}

Os estudos de neuroimagem investigando o uso de antipsicóticos na esquizofrenia indicam que são necessárias ocupações de receptores D2 superiores a $65 \%$ ou $70 \%$ para se obter resposta clínica. Entretanto, a janela terapêutica é estreita, pois quando a ocupação supera $78 \%$, a chance de se desenvolver tanto sintomas extrapiramidais como sintomas depressivos é alta. Doses habituais de antipsicóticos atípicos obtêm resposta clínica com ocupações estriatais inferiores a $75 \%$, portanto, apresentam chance muito menor de desencadear sintomas extrapiramidais ou depressivos. Estudos recentes sugerem que isto é possível devido à seletividade por receptores D2 de regiões límbicas dos antipsicóticos atípicos.

Os achados de aumento da atividade da enzima dopadescarboxilase, juntamente com o aumento da liberação de dopamina induzido por anfetamina e a elevação dos níveis de dopamina na fenda sináptica, representam um forte indício de elevação da liberação fásica de dopamina na esquizofrenia. Esses achados são, certamente, uma das mais importantes descobertas da fisiopatologia da esquizofrenia nos últimos anos. Apesar dos estudos confirmarem uma disfunção dopaminérgica na esquizofrenia, eles mostram que essa alteração não é primária deste sistema. ${ }^{23}$ Provavelmente existem disfunções nos sistemas responsáveis pela regulação dopaminérgica, tais como o sistema glutamatérgico. ${ }^{24} \mathrm{~A}$ descoberta de novos ligantes permitirá o estudo de sistemas de neurotransmissão ainda não estudados in vivo. ${ }^{25}$

Fonte de financiamento: Rodrigo A Bressan é financiado por uma grant de pesquisa sem restrições da Jansen Pharmaceutica

\section{Referências}

1. Rice DP. The economic impact of schizophrenia. J Clin Psychiatry 1999;60(1):4-6.

2. Nemeroff $\mathrm{CB}$, Kilts $\mathrm{CD}$, Berns GS. Functional brain imaging: twenty-first century phrenology or psychobiological advance of the millennium. Am J Psychiatry 1999;155:671-3.

3. Seibyl JP. Development of SPET as a tool for neuropsychopharmacological research. In: Neuroimaging. Cambridge University Press; 1995. p. 59-80.

4. Bigliani V, Pilowsky LS. In vivo neuropharmacology of schizophrenia. Br J Psychiatry 1999;174(38):23-33.

5. Carlsson A. The current status of the dopamine hypothesis of schizophrenia. Neuropsychopharmacol 1988;1:179-86.

6. Abi-Dargham A, Rodenhiser J, Prinz D, Zea-Ponce Y, Gil R, Kegeles LS, et al. Increased baseline occupancy of D2 receptors by dopamine in schizophrenia. Proc Natl Acad Sci 2000;97(14):8104-09.
7. Laruelle M, Abi-Dargham A, van Dyck CH, Gil R, D'Souza C, Erdos $\mathrm{J}$, et al. Single photon emission computerized tomography imaging of amphetamine-induced dopamine release in drug-free schizophrenic subjects. Proc Natl Acad Sci USA 1996;93(1):9235-40.

8. Abi-Dargham A, Gil R, Krystal J, Baldwin RM, Seibyl JP, Bowers M, et al. Increased striatal dopamine transmission in schizophrenia: confirmation in a second cohort. Am J Psychiatry 1998;155(6):761-7.

9. Breier A, Su TP, Saunders R, Carson RE, Kolachana BS. Schizophrenia is associated with elevated amphetamine-induced synaptic dopamine concentrations: evidence from a novel positron emission tomography method. Proc Nat Acad Sci USA 1997;94(6):2569-74.

10. Pilowsky LS, Costa DC, Ell PJ, Murray RM, Verhoeff NPLG, Kerwin RW. Antipsychotic medication, D2 dopamine receptor blockade and clinical response - a 123I-IBZM SPET study. Psychol Med 1993;23(3):791-7.

*Bressan RA, Costa DC, Jones HM, Ell PJ, Pilowsky LS. Typical antipsychotic drugs - D2 receptor blockade and depressive symptoms in schizophrenia. Schizophr Res. No prelo. 
11. Pilowsky LS, Costa DC, Ell PJ, Murray RM, Verhoeff NP. Clozapine, single photon emission tomography, and the D2 dopamine receptor. Lancet 1992;340(8813):199-202.

12. Farde L, Nordstrom AL, Wiesel FA, Pauli S, Halldin C, Sedvall G. Positron emission tomographic analysis of central D1 and D2 dopamine receptor occupancy in patients treated with classical neuroleptics and clozapine. Relation to extrapyramidal side effects. Arch Gen Psychiatry 1992;49(7):538-44.

13. Kapur S, Zipursky RB, Jones C, Remington GJ, Houle S. Relationship between dopamine D2 occupancy, clinical response, and side effects: a double-blind PET study of first-episode schizophrenia. Am J Psychiatry 2000;157:514-20.

14. Nordstrom AL, Farde L. Plasma prolactin and central D2 receptor occupancy in antipsychotic drug-treated patients. J Clin Psychopharmacol 1998;18(4):305-10.

15. Farde L, Nordstrom AL, Nyberg S, Halldin C, Sedvall G. D1-, D2-, and 5-HT2-receptor occupancy in clozapine-treated patients. J Clin Psychiatry 1994;55:67-9.

16. Kapur S, Zipursky R, Jones C, Shammi CS, Remington G, Seeman P. A positron emission tomography study of quetiapine in schizophrenia: a preliminary finding of an antipsychotic effect with only transiently high dopamine D2 receptor occupancy. Arch Gen Psychiatry 2000;57(6):553-9.

17. Pilowsky LS, Busatto GF, Taylor M, Costa DC, Sharma T, Sigmundsson T, Ell PJ, Nohria V, Kerwin RW. Dopamine D2 receptor occupancy in vivo by the novel atypical antipsychotic olanzapine - a 123I IBZM single photon emission tomography (SPET) study. Psychopharmacol 1996;124(1-2):148-53.
18. Busatto GF, Pilowsky LS, Ell PJ, Costa DC, Verhoeff NP, Kerwin RW. Dopamine D2 receptor occupancy in vivo and response to the new antipsychotic risperidone. $\mathrm{Br} \quad \mathrm{J}$ Psychiatry $1993 ; 163: 833-4$.

19. Bigliani V, Mulligan RS, Acton PD, Visvikis D, Ell PJ, Stephenson C, Kerwin RW, Pilowsky LS. In vivo occupancy of striatal and temporal cortical D2/D3 dopamine receptors by typical antipsychotic drugs. [123I] epidepride single photon emission tomography (SPET) study. Br J Psychiatry 1999;175:231-8.

20. Pilowsky LS, Mulligan RS, Acton PD, Ell PJ, Costa DC, Kerwin RW. Limbic selectivity of clozapine. Lancet 1997;350(9076):490-1.

21. Bigliani V, Mulligan RS, Acton PD, Ohlsen RI, Pike VW, Ell PJ, Gacinovic S, Kerwin RW, Pilowsky LS. Striatal and temporal cortical D2/D3 receptor occupancy by olanzapine and sertindole in vivo: a [123I] epidepride SPET study. Psychopharmacol 2000;150(2):132-40.

22. Stephenson CM, Bigliani V, Jones HM, Kerwin RW, Pilowsky LS, Mulligan RS, Visvikis D, Ell PJ, Acton PD. Striatal and extrastriatal $\mathrm{D}(2) / \mathrm{D}(3)$ dopamine receptor occupancy by quetiapine in vivo: a [123I] epidepride SPET study. Br J Psychiatry 2000; $177: 408-15$.

23. Laruelle M, Abi-Dargham, Gil R, Kegeles L, Innis R. Increased dopaminergic transmission in schizophrenia: relationship to illness phases. Biol Psychiatry 1999;46:56-72.

24. Moore H, Wet AR, Grace AA. The regulation of forebrain dopamine transmission: relevance to the pathology and psychopathology of schizophrenia. Biol Psychiatry 1999;46:40-55.

25. Bressan RA, Pilowsky LS. Imaging the glutamatergic system in vivo - relevance to schizophrenia. Eur J Nucl Med 2000;27:1723-31

Correspondência: Rodrigo A Bressan

De Crespigny Park, SE5 8AF, London, UK

Tel.: (00xx44) (20) 78480807 - Fax: (00xx44) (20) 78480051 - E-mail: r.bressan@iop.kcl.ac.uk 
Rev Bras Psiquiatr 2001;23(Supl I):61-4

Neuroimagem de receptores D2 de dopamina na esquizofrenia - p. 46-49

Figura - Ocupação de receptores D2 no striatum por antipsicóticos utilizando ${ }^{123}$ IBZM scans e Spet

A) Voluntário sadio

(sem medicação)

B) Paciente tomando

antipsicótico atípico

C) Paciente tomando antipsicótico típico

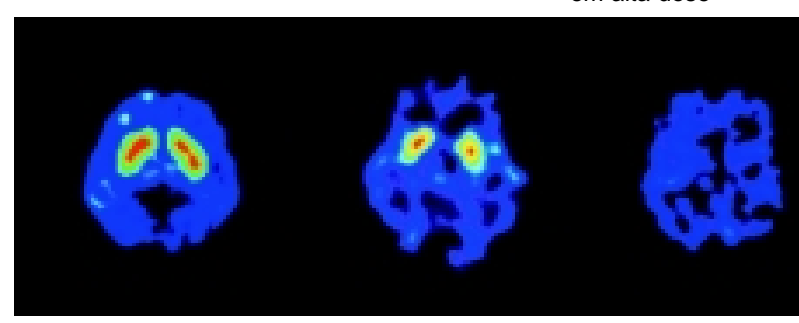

A) $0 \%$

B) $78 \%$

C) $100 \%$ 